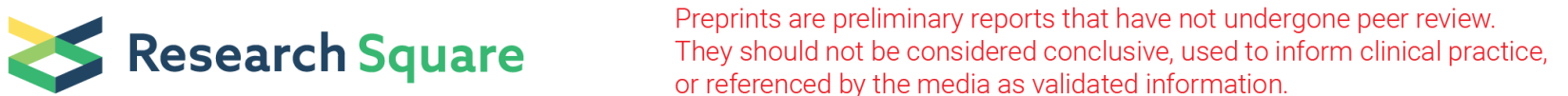 \\ Changes in the Prevalence of Hyperuricemia in Clients of Health Examination in Eastern China, 2009 to 2019
}

\section{Dunmin She}

Northern Jiangsu People's Hospital Affiliated to Yangzhou University

\section{Yongliang Wang}

Yangzhou University

Jing Liu

Dalian Medical University

Na Luo

Northern Jiangsu People's Hospital Affiliated to Yangzhou University

\section{Shangyong Feng}

Northern Jiangsu People's Hospital Affiliated to Yangzhou University

Ying Li

Northern Jiangsu People's Hospital Affiliated to Yangzhou University

Jin Xu

Northern Jiangsu People's Hospital Affiliated to Yangzhou University

Shichun Xie

Northern Jiangsu People's Hospital Affiliated to Yangzhou University

Yan Zhu

Northern Jiangsu People's Hospital Affiliated to Yangzhou University

Ying Xue

Tongji University

Zhenwen Zhang ( $\nabla$ yzzzw@medmail.com.cn )

Northern Jiangsu People's Hospital Affiliated to Yangzhou University

\section{Research Article}

Keywords: Prevalence, Hyperuricemia, Serum uric acid level, Age, Body mass index

Posted Date: January 14th, 2022

DOl: https://doi.org/10.21203/rs.3.rs-1204980/v1

License: @ (1) This work is licensed under a Creative Commons Attribution 4.0 International License. Read Full License 


\section{Abstract}

Background: With the continuous improvement of people's living standards, the incidence of hyperuricemia (HUA) is increasing globally. The prevalence of HUA ranged in terms of region, race, and age. This study aims to investigate the changes in the prevalence of HUA in clients of health examination in Eastern China between 2009 and 2019.

Methods: Chinese men and women aged 20-79 years $(n=4847$ in the 2009 cohort and $n=12188$ in 2019) who had received health examinations were enrolled. Serum uric acid (UA) levels and biochemical parameters, including fasting blood-glucose (FBG), triglyceride $(\mathrm{TG})$, total cholesterol (CHOL), high-density lipoprotein ( $\mathrm{HDL}$ ), low-density lipoprotein (LDL), creatinine (Cr) and blood urea nitrogen (BUN) were evaluated. The prevalence of HUA in different age groups were measured, and the correlation of biochemical parameters with the onset of HUA were analyzed.

Results: The prevalence of HUA was 18.7\% in the 2019 cohort, which was significantly higher than that in 2009 (13.3\%). In females, the prevalence of HUA was significantly higher in 2019 than 2009 for age groups of 20-29 and 30-39 years. In male population, 2019 cohort had significantly higher age-specific prevalence for all age groups than 2009 cohort. Young men aged 20-29 years became the main population of HUA in the 2019 male cohort, whereas middle-aged men aged 40-49 years had the highest prevalence of HUA in the 2009 male cohort. The prevalence rates of HUA in all BMI groups in 2019 cohort were significantly higher than those in 2009 cohort. Spearmen's correlation analysis and Logistic regression analysis indicated that BMI was positively correlated with the onset of HUA. The receiver-operating characteristic curve (ROC) analysis showed BMI $24.48 \mathrm{~kg} / \mathrm{m} 2$ and BMI $23.84 \mathrm{~kg} / \mathrm{m} 2$ displayed good capacities to discriminate the population with HUA from those without HUA in 2009 and 2019 cohort, respectively.

Conclusions: In recent 10 years, the prevalence of HUA was increased rapidly in Chinese adults, especially in males. Young men aged 2029 years in the 2019 cohort replaced the middle-aged males (40-49 years old) in the 2009 cohort, and became the main population of male HUA in the 2019 cohort. BMI was positively correlated with HUA, and might be a potential risk factors to predict the onset of HUA.

\section{Background}

Uric acid (2,6,8 trioxypurine-C5H4N4O3, UA) is the final enzymatic product of purine metabolism in the body [1]. It can come from the human body or from the decomposition and metabolism of purines in food. Hyperuricemia (HUA) occurs due to increased UA production, impaired renal UA excretion, or a combination of both [2]. HUA is defined as blood uric acid concentration equals to and greater than $420 \mu \mathrm{mol} / \mathrm{L}$ in males and $360 \mu \mathrm{mol} / \mathrm{L}$ in females [3]. Elevated blood UA levels not only induce gouty arthritis, urolithiasis and UA nephropathy [4-6], but are also associated with type 2 diabetes, hypertension, cardiovascular disease (CVD) and chronic kidney disease (CKD) [7-10].

The prevalence of HUA ranged from $2.6-40 \%$ in terms of region, race, and research method [11]. The prevalence of HUA in some developed countries, such as USA (21.4\%) and Japan (25.8\%), is much higher than that of developing countries including Saudi Arabia (8.4\%), Thailand (10.6\%) and Turkey $(12.1 \%)[12,13]$. Due to the vast territory of China, the prevalence of HUA varies significantly in different geographic regions. Generally, HUA is more common in cities than in rural regions, and higher in southern areas than in northern areas [14]. The prevalence of HUA in some economically developed regions of China, such as Guangdong (19.2\%) [15], Beijing (17.9\%) [16], and Hainan (25.1\%) [17], is similar to that of developed countries [12, 13]. However, in some underdeveloped areas of China, the prevalence of HUA is much lower than those of developed regions in China during the same period [18, 19]. A previous study showed that the combined prevalence of HUA in rural areas was $11.7 \%$ in China [3]. A population-based study reported that the prevalence of HUA among the middle-aged and elderly people in Tibet was only $2.05 \%$ [20]. Although recent studies have described the prevalence of HUA in China [21-24], there is still a lack of research on the changing trend of the prevalence of HUA in China over the past 10 years, especially the characteristics of the prevalence of HUA among people of different genders and ages. The aims of this study were to compare the prevalence of HUA in different age groups in the 2009 cohort and 2019 cohort, and to analyze the correlations between HUA biochemical parameters and the onset of HUA in Chinese adults.

\section{Methods}

\subsection{Study population}

Participants were Chinese men and women who had underwent health examinations in Northern Jiangsu People's Hospital ( $n=4847$ in the 2009 cohort and $n=12188$ in 2019). The inclusion criteria were individuals aged 20-79 years and those who consented to participate 
in this study. The exclusion criteria were those who had missing data and who were underweight $(\mathrm{BMl}<18.5 \mathrm{~kg} / \mathrm{m} 2)$. All participants agreed to participate in the study and the study was approved by the Ethical Committee of Northern Jiangsu People's Hospital.

\subsection{Data collection}

The following data were collected: date of birth/death, gender, personal medical history, family history, body height (BH, $\mathrm{m})$, body weight (BW, kg), systolic blood pressure (SBP, $\mathrm{mmHg}$ ), diastolic blood pressure ( $\mathrm{DBP}, \mathrm{mmHg}$ ) and blood biochemical tests. BH, BW, and BP were measured according to standardized protocols. All blood tests were performed at the clinical laboratory of Northern Jiangsu Hospital. Blood biochemical tests included serum urid acid level (UA reference range, 143-339 $\mu \mathrm{mol} / \mathrm{L}$ ), fasting blood-glucose (FBG, 3.9-6.1 $\mathrm{mmol} / \mathrm{L})$, triglyceride $(\mathrm{TG},<1.7 \mathrm{mmol} / \mathrm{L})$, total cholesterol $(\mathrm{CHOL},<5.17 \mathrm{mmol} / \mathrm{L})$, high-density lipoprotein ( $\mathrm{HDL}, 1.29-1.55 \mathrm{mmol} / \mathrm{L})$, lowdensity lipoprotein $(\mathrm{LDL},<3.37 \mathrm{mmol} / \mathrm{L})$, creatinine $(\mathrm{Cr}, 44-133 \mu \mathrm{mol} / \mathrm{L})$, and blood urea nitrogen $(\mathrm{BUN}, 3.1-8.0 \mathrm{mmol} / \mathrm{L})$.

\subsection{Definition and grouping}

HUA was defined as serum UA $\geq 420.0 \mu \mathrm{mol} / \mathrm{L}$ in males and $\geq 360 \mu \mathrm{mol} / \mathrm{L}$ in female subjects [25]. Body mass index $\left(B M I, \mathrm{~kg} / \mathrm{m}^{2}\right) \mathrm{was}$ calculated using the following formula: BMI $\left(\mathrm{kg} / \mathrm{m}^{2}\right)=$ weight $(\mathrm{kg}) /$ height $^{2}\left(\mathrm{~m}^{2}\right)$. BMI was divided into normal weight $\left(18.5 \mathrm{~kg} / \mathrm{m}^{2} \leq\right.$ $\left.\mathrm{BMI}<24.0 \mathrm{~kg} / \mathrm{m}^{2}\right)$, overweight $\left(24.0 \mathrm{~kg} / \mathrm{m}^{2} \leq \mathrm{BMI}<28.0 \mathrm{~kg} / \mathrm{m}^{2}\right)$, and obesity $\left(\mathrm{BMI} \geq 28.0 \mathrm{~kg} / \mathrm{m}^{2}\right)$ according to the revised Asia-Pacific BMI criteria published by the World Health Organization (WHO) [26]. All the participants were divided into six age categories: 20-29 years, 30-39 years, 40-49 years, 50-59 years, 60-69 years and 70-79 years old.

\subsection{Statistical analysis}

Statistical package for social sciences (SPSS) 25.0 was used for the statistical analysis in this study. All continuous variables were presented as mean \pm standard deviation. Chi-square test was used to compare categorical variables, and independent-Samples T-Test was for continuous variables between two groups. Spearman correlations and binary logistic regression analysis were performed to explore the risk factors of the onset of HUA. Receiver-operating characteristic (ROC) curve analysis was used to determine the cut-off value, sensitivity and specificity of the risk factors which can potentially predict the onset of HUA. Tests were two-sided and a $p$-value < 0.05 was considered significant.

\section{Results}

\subsection{Clinical characteristics of participants in the 2009 and 2019 cohorts}

Clinical characteristics of participants in the 2009 and 2019 cohorts were shown in Table 1. In the 2009 cohort, 1682 participants were females and 3165 were males, while 3852 females and 8336 males were included in the 2019 cohort. The participants in the 2009 cohort were significantly older than those in the 2019 cohort ( $46.6 \pm 13.9$ years vs. $45.3 \pm 13.3$ years, $p<0.01)$. BMI, levels of DBP, FBG, $\mathrm{CHOL}$ and BUN were significantly lower in the 2019 cohort than those in the 2009 cohort, whereas levels of SBP, TG, HDL, LDL and Cr were significantly higher in 2019 cohort than those in the 2009 cohort. In addition, serum UA level of participants in the 2019 cohort was significantly higher than that in the 2009 cohort(339.4 $\pm 85.4 \mu \mathrm{mol} / \mathrm{L}$ vs $314.5 \pm 80.8 \mu \mathrm{mol} / \mathrm{L})$.

Table1 Baseline characteristics of participants enrolled in 2009 and 2019 


\begin{tabular}{|c|c|c|c|c|c|c|}
\hline \multirow[t]{3}{*}{ Variables } & \multicolumn{3}{|l|}{2009 cohort } & \multicolumn{3}{|l|}{2019 cohort } \\
\hline & HUA & Non- HUA & Total & HUA & Non- HUA & Total \\
\hline & $\triangle n=538 \rrbracket$ & $\nabla n=4309 \rrbracket$ & $\bigotimes n=4847 \rrbracket$ & $\bigotimes n=2282 \bigotimes$ & $\nabla n=9906 \rrbracket$ & $\bigotimes n=12188 \rrbracket$ \\
\hline $\mathrm{F} / \mathrm{M}$ & $64 / 474$ & $1618 / 2691^{*}$ & $1682 / 3165$ & $214 / 2068$ & $3638 / 6268^{*}$ & $3852 / 8336$ \\
\hline Mean age & $49.3 \pm 13.9$ & $46.3 \pm 13.9^{*}$ & $46.6 \pm 13.9$ & $44.5 \pm 13.6$ & $45.5 \pm 13.2^{*}$ & $45.3 \pm 13.3^{\triangle}$ \\
\hline BMI (kg/m2) & $25.7 \pm 2.7$ & $23.4 \pm 3.0^{\star}$ & $23.9 \pm 3.0$ & $25.7 \pm 3.1$ & $23.7 \pm 3.1^{*}$ & $23.6 \pm 3.2^{\triangle}$ \\
\hline $\mathrm{SBP}(\mathrm{mmHg})$ & $128.5 \pm 16.6$ & $121.0 \pm 22.9^{*}$ & $121.8 \pm 22.4$ & $136.3 \pm+17.1$ & $131.0 \pm 20.7^{\star}$ & $132.0 \pm 20.2^{\triangle}$ \\
\hline $\mathrm{DBP}(\mathrm{mmHg})$ & $86.5 \pm 10.8$ & $80.2 \pm 10.8^{\star}$ & $80.9 \pm 11.0$ & $83.5 \pm 12.1$ & $78.9 \pm 12.1^{*}$ & $79.8 \pm 12.2^{\triangle}$ \\
\hline FBG (mmol/L) & $5.67 \pm 1.12$ & $5.35 \pm 1.21^{*}$ & $5.39 \pm 1.21$ & $5.24 \pm 1.16$ & $5.23 \pm 1.41$ & $5.23 \pm 1.36^{\triangle}$ \\
\hline TG $(\mathrm{mmol} / \mathrm{L})$ & $2.53 \pm 1.93$ & $1.51 \pm 1.31^{\star}$ & $1.62 \pm 1.43$ & $2.39 \pm 2.03$ & $1.60 \pm 1.43^{*}$ & $1.75 \pm 1.59 \triangle$ \\
\hline $\mathrm{TC}(\mathrm{mmol} / \mathrm{L})$ & $5.00 \pm 0.99$ & $4.52 \pm 0.85^{\star}$ & $4.58 \pm 0.88$ & $4.71 \pm 0.86$ & $4.49 \pm 0.85^{\star}$ & $4.53 \pm 0.85^{\triangle}$ \\
\hline $\mathrm{HDL}$ (mmol/L) & $1.11 \pm 0.25$ & $1.25 \pm 0.32^{*}$ & $1.24 \pm 0.32$ & $1.10 \pm 0.29$ & $1.31 \pm 0.37^{\star}$ & $1.27 \pm 0.36^{\triangle}$ \\
\hline LDL (mmol/L) & $2.71 \pm 0.72$ & $2.45 \pm 0.65^{\star}$ & $2.48 \pm 0.66$ & $2.70 \pm 0.73$ & $2.50 \pm 0.71^{*}$ & $2.53 \pm 0.71^{\triangle}$ \\
\hline BUN (mmol/L) & $5.89 \pm 1.47$ & $5.38 \pm 1.42^{*}$ & $5.44 \pm 1.43$ & $5.36 \pm 1.43$ & $5.04 \pm 1.28^{\star}$ & $5.10 \pm 1.29 \triangle$ \\
\hline $\operatorname{Cr}(\mu \mathrm{mol} / \mathrm{L})$ & $79.3 \pm 14.8$ & $67.8 \pm 18.5^{\star}$ & $69.1 \pm 18.5$ & $93.7 \pm 25.5$ & $81.6 \pm 18.8^{\star}$ & $83.9 \pm 20.8^{\triangle}$ \\
\hline $\mathrm{UA}(\mu \mathrm{mol} / \mathrm{L})$ & $457.6 \pm 50.1$ & $297.1 \pm 64.6^{*}$ & $314.5 \pm 80.8$ & $464.6 \pm 53.3$ & $310.6 \pm 62.3^{*}$ & $339.4 \pm 85.4 \triangle$ \\
\hline
\end{tabular}

${ }^{*} P<0.01$ comparing participants with hyperuricemia to those with non-hyperuricemia within each cohort

$\triangle P<0.01$ comparing participants between the 2009 cohort and the 2019 cohort

$U A$ uric acid, $B M /$ body mass index, $S B P$ systolic blood pressure, $D B P$ Diastolic blood pressure, $F B G$ fasting blood glucose, $T G$ triglycerides, $T C$ total cholesterol, $H D L$ high density lipoprotein, $L D L$ low density lipoprotein, $B U N$ blood urea nitrogen, $C r$ creatinine

\subsection{The prevalence of HUA in all age groups in the 2009 and 2019 cohorts}

In Table 2 and Fig. 1, we presented the prevalence of HUA and serum UA levels in all age groups in the 2009 and 2019 cohorts. In the 2019 cohort, the total prevalence of HUA was $18.3 \%$, which was significantly higher than that in the 2009 cohort $(13.3 \%)(p<0.01)$. HUA was more common in males than in females both in the 2009 cohort $(17.3 \%$ vs. $4.5 \%, p<0.01)$ and 2019 cohort $(26.4 \%$ vs. $7.5 \% p$ $<0.01)$.

In females, the prevalence of HUA in participants aged 20-29 and 30-39 years in the 2019 cohort was relatively higher than that in the 2009 cohort $(p<0.01)$. There was no difference in the prevalence of HUA in participants aged 40-49, 50-59, 60-69 and 70-79 years between 2009 cohort and 2019 cohort. In the 2009 and 2019 cohort, the prevalence of HUA in elderly females ( $\geq 60$ years old) was significantly higher than that of young women and middle-aged women (20-59 years old), and the prevalence of HUA reached its peak at the age of 70-79.

For males, the prevalence of HUA in all age groups in 2019 cohort was significantly higher than that in the 2009 cohort $(p<0.01)$. The prevalence of HUA among young men (20-29 and 30-39 years old) was significantly higher than those of the middle-aged and elderly men in the 2019 cohort. The prevalence of HUA in males aged 20-29 years in the 2019 cohort (31.92\%) was nearly triple that of the same age group in the 2009 cohort (10.04\%), while the prevalence of males aged 30-39 years in the 2019 cohort (29.94\%) was more than twice that of the same age group in 2009 (14.21\%). In the 2019 cohort, young males aged 20-29 years had the highest prevalence of HUA, while the HUA prevalence reached its peak in the middle-aged males (aged 40-49 years) in the 2009 cohort.

\subsection{Serum UA levels in all age groups in the 2009 and 2019 cohorts}


The change trend of serum UA levels of all age categories was similar to the change in the prevalence of HUA in both men and women. Serum UA levels of both males and females in the 2019 cohort were significantly higher than those in the 2009 cohort (males: $373 \pm 76.2$ vs. $349 \pm 70.0 \mu \mathrm{mol} / \mathrm{L}, p<0.01$; females: $267 \pm 53.2 \mathrm{vs} .250 \pm 55.2 \mu \mathrm{mol} / \mathrm{L}, p<0.01$ ). The total UA levels in males were dramatically higher than those in females both in the 2009 and 2019 cohort (in 2009: $349 \pm 70.0$ vs. $250 \pm 55.2 \mu \mathrm{mol} / \mathrm{L}, p<0.01$; in 2019: $373 \pm 76.2$ vs. $267 \pm 53.2 \mu \mathrm{mol} / \mathrm{L}, p<0.01)$. Serum UA levels of all age groups in males were also obviously higher than those in females both in the 2009 cohort and 2019 cohort. Serum UA levels in young and middle-age female participants (20-29, 30-39, 40-49 and 50-59 years old) in the 2019 cohort were significantly higher than those in the 2009 cohort. However, there was no difference in the UA levels of elderly females (60-69 and 70-79 age group) between the 2019 cohort and 2009 cohort. In males, UA levels of all age groups in the 2019 cohort were significantly higher than those in the 2009 cohort. (Table 2 and Fig. 1)

\subsection{The prevalence of HUA in different BMI groups in the 2009 and 2019 cohort}

All participants were divided into three BMI groups, including normal weight $\left(B M l<24 \mathrm{~kg} / \mathrm{m}^{2}\right)$, overweight $\left(24 \mathrm{~kg} / \mathrm{m}^{2} \leq \mathrm{BMl}<28 \mathrm{~kg} / \mathrm{m}^{2}\right)$ and obesity $\left(\mathrm{BMl} \geq 28 \mathrm{~kg} / \mathrm{m}^{2}\right)$. The prevalence of HUA in different BMI groups in the 2009 and 2019 cohort were presented in Table 3 and Fig. 1. In males, the prevalence of HUA in all BMI groups in the 2019 cohort were significantly higher than those in the 2009 cohort. The prevalence of HUA in obese men was markedly higher than that of males with normal weight and overweight both in the 2009 and 2019 cohort. We found that the prevalence of HUA in males in both 2009 and 2019 cohort was dramatically increased along with the elevated BMI value. However, this change trend was not significant in females.

Table 3

Prevalence of HUA in 2009 and 2019 cohort in BMI category

\begin{tabular}{|c|c|c|c|c|c|c|c|}
\hline & & \multicolumn{2}{|c|}{$\begin{array}{l}\text { Normal weight } \\
\left(\mathrm{BMl}<24 \mathrm{~kg} / \mathrm{m}^{2}\right)\end{array}$} & \multicolumn{2}{|c|}{$\begin{array}{l}\text { Overweight } \\
\left(24 \mathrm{~kg} / \mathrm{m}^{2} \leq \mathrm{BM}<28 \mathrm{~kg} / \mathrm{m}^{2}\right)\end{array}$} & \multicolumn{2}{|c|}{$\begin{array}{l}\text { Obese } \\
\left(\mathrm{BMI} \geq 28 \mathrm{~kg} / \mathrm{m}^{2}\right)\end{array}$} \\
\hline & & $\mathrm{F}$ & M & $\mathrm{F}$ & M & $\mathrm{F}$ & M \\
\hline \multirow[t]{2}{*}{ Prevalence of HUA } & 2009 cohort & $1.73 \%$ & $7.59 \% \triangle$ & $10.54 \% \$$ & $18.47 \% \triangle \$$ & $19.51 \% \$$ & $28.13 \% \triangle \$ \&$ \\
\hline & 2019 cohort & $5.15 \%^{* *}$ & $15.83 \%^{* \star} \triangle$ & $6.75 \%^{*}$ & $27.43 \%{ }^{* \star} \triangle \$$ & $7.19 \%^{*}$ & $40.67 \%{ }^{* \star} \triangle \$ \&$ \\
\hline \multicolumn{8}{|c|}{${ }^{*} p<0.05$ comparing participants with those in 2009 cohort } \\
\hline \multicolumn{8}{|c|}{${ }^{\star *} p<0.01$ comparing participants with those in 2009 cohort } \\
\hline \multicolumn{8}{|c|}{$\triangle p<0.01$ comparing male participants with females } \\
\hline \multicolumn{8}{|c|}{$\$ p<0.01$ comparing with participants with normal weight } \\
\hline \multicolumn{8}{|c|}{$\& p<0.01$ comparing with participants with overweight } \\
\hline$B M /$ body mass ind & HUA hyperur & emia & & & & & \\
\hline
\end{tabular}

The prevalence of HUA in females with normal weight in the 2019 cohort was significantly higher than that in the 2009 cohort (5.15\% vs. $1.73 \%, p<0.01)$. However, the prevalence of HUA in females with obesity and overweight was obviously lower in the 2019 cohort compared with that in the 2009 cohort ( $6.75 \%$ vs. $10.54 \%$ in obese group, $p<0.05 ; 7.19 \%$ vs. $19.51 \%$ in overweight group, $p<0.05)$. In addition, the prevalence of HUA of obese and overweight females in the 2009 cohort were higher than that of females with normal weight. There was no significant difference in the prevalence of HUA between obese and overweight females in the 2009 cohort. In the 2019 cohort, there was no significant difference in the prevalence of HUA of females among three BMI groups.

\subsection{Risk factors for the onset of HUA}

The relationship of biochemical parameters and the onset of HUA using spearman's correlation analysis was shown in Table 4. BMI, BH, BW, FBG, TG, CHOL, LDL, Cr and BUN were positively associated with serum UA levels and the onset of HUA in the overall participants in 
both 2009 and 2019 cohort, while gender and HDL were negatively related to UA levels and the onset of HUA. In the 2009 cohort, age was positively correlated with serum UA levels and the onset of HUA, whereas in the 2019 cohort, gender was negatively associated with the onset of HUA, and was not correlated with serum UA levels.

Table 4

Correlations of UA levels and HUA with related variables in cohort 2009 and 2019

\begin{tabular}{|c|c|c|c|c|c|c|c|c|c|c|c|c|c|}
\hline & & Age & BMI & $\mathrm{BH}$ & BW & gender & FBG & TG & $\mathrm{TC}$ & $\mathrm{HDL}$ & LDL & $\mathrm{Cr}$ & BUN \\
\hline \multirow{4}{*}{$\begin{array}{l}\text { UA } \\
\text { level }\end{array}$} & \multirow{2}{*}{$\begin{array}{l}2009 \\
\text { cohort }\end{array}$} & 0.134 & 0.458 & 0.482 & 0.570 & -0.617 & 0.191 & 0.458 & 0.187 & -0.333 & 0.196 & 0.239 & 0.596 \\
\hline & & $\begin{array}{l}(p< \\
0.01)\end{array}$ & $\begin{array}{l}(p< \\
0.01)\end{array}$ & $\begin{array}{l}(p< \\
0.01)\end{array}$ & $\begin{array}{l}(p< \\
0.01)\end{array}$ & $\begin{array}{l}(p< \\
0.01)\end{array}$ & $\begin{array}{l}(p< \\
0.01)\end{array}$ & $\begin{array}{l}(p< \\
0.01)\end{array}$ & $\begin{array}{l}(p< \\
0.01)\end{array}$ & $\begin{array}{l}(p< \\
0.01)\end{array}$ & $\begin{array}{l}(p< \\
0.01)\end{array}$ & $\begin{array}{l}(p< \\
0.01)\end{array}$ & $\begin{array}{l}(p< \\
0.01)\end{array}$ \\
\hline & \multirow{2}{*}{$\begin{array}{l}2019 \\
\text { cohort }\end{array}$} & 0.003 & 0.396 & 0.474 & 0.562 & -0.610 & 0.099 & 0.416 & 0.131 & -0.426 & 0.177 & 0.585 & 0.197 \\
\hline & & $\begin{array}{l}(p= \\
0.756)\end{array}$ & $\begin{array}{l}(p< \\
0.01)\end{array}$ & $\begin{array}{l}(p< \\
0.01)\end{array}$ & $\begin{array}{l}(p< \\
0.01)\end{array}$ & $\begin{array}{l}(p< \\
0.01)\end{array}$ & $\begin{array}{l}(p< \\
0.01)\end{array}$ & $\begin{array}{l}(p< \\
0.01)\end{array}$ & $\begin{array}{l}(p< \\
0.01)\end{array}$ & $\begin{array}{l}(p< \\
0.01)\end{array}$ & $\begin{array}{l}(p< \\
0.01)\end{array}$ & $\begin{array}{l}(p< \\
0.01)\end{array}$ & $\begin{array}{l}(p< \\
0.01)\end{array}$ \\
\hline \multirow{4}{*}{$\begin{array}{l}\text { onset } \\
\text { of } \\
\text { HUA }\end{array}$} & \multirow[t]{2}{*}{2009 cohort } & 0.074 & 0.242 & 0.125 & 0.237 & -0.169 & 0.146 & 0.263 & 0.153 & -0.152 & 0.119 & 0.112 & 0.235 \\
\hline & & $\begin{array}{l}(p< \\
0.01)\end{array}$ & $\begin{array}{l}(p< \\
0.01)\end{array}$ & $\begin{array}{l}(p< \\
0.01)\end{array}$ & $\begin{array}{l}(p< \\
0.01)\end{array}$ & $\begin{array}{l}(p< \\
0.01)\end{array}$ & $\begin{array}{l}(p< \\
0.01)\end{array}$ & $\begin{array}{l}(p< \\
0.01)\end{array}$ & $\begin{array}{l}(p< \\
0.01)\end{array}$ & $\begin{array}{l}(p< \\
0.01)\end{array}$ & $\begin{array}{l}(p< \\
0.01)\end{array}$ & $\begin{array}{l}(p< \\
0.01)\end{array}$ & $\begin{array}{l}(p< \\
0.01)\end{array}$ \\
\hline & \multirow{2}{*}{$\begin{array}{l}2019 \\
\text { cohort }\end{array}$} & -0.034 & 0.242 & 0.193 & 0.292 & -0.229 & 0.066 & 0.269 & 0.104 & -0.237 & 0.101 & 0.285 & $0-.092$ \\
\hline & & $\begin{array}{l}(p< \\
0.01)\end{array}$ & $\begin{array}{l}(p< \\
0.01)\end{array}$ & $\begin{array}{l}(p< \\
0.01)\end{array}$ & $\begin{array}{l}(p< \\
0.01)\end{array}$ & $\begin{array}{l}(p< \\
0.01)\end{array}$ & $\begin{array}{l}(p< \\
0.01)\end{array}$ & $\begin{array}{l}(p< \\
0.01)\end{array}$ & $\begin{array}{l}(p< \\
0.01)\end{array}$ & $\begin{array}{l}(p< \\
0.01)\end{array}$ & $\begin{array}{l}(p< \\
0.01)\end{array}$ & $\begin{array}{l}(p< \\
0.01)\end{array}$ & $\begin{array}{l}(p< \\
0.01)\end{array}$ \\
\hline \multicolumn{14}{|c|}{$\begin{array}{l}\text { UA uric acid, } B M / \text { body mass index, } B H \text { body height, } B W \text { body weight, } F B G \text { fasting blood glucose, } T G \text { triglycerides, } T C \text { total } \\
\text { cholesterol, } H D L \text { high density lipoprotein, } L D L \text { low density lipoprotein, } C r \text { creatinine, }\end{array}$} \\
\hline
\end{tabular}

To further investigate the associations between the onset of HUA and BMI, unadjusted and multivariate adjusted logistic regression analyses were performed. BMI was significantly positively associated with HUA in the 2009 cohort (OR: 1.212, 95\% confidence interval (Cl): 1.133-1.296, $p<0.01$ ) and 2019 cohort (OR: 1.200, 95\% Cl: 1.161-1.241, $p<0.01$ ). Those associations also persisted after adjustment for age, gender, BUN, Cr, FBG, TG, CHOL, HDL and LDL (2009 cohort: OR: 1.136, 95\% Cl: 1.054-1.224, $p<0.01 ; 2019$ cohort: OR: $1.102,95 \% \mathrm{Cl}: 1.063-1.43, p<0.01)$. ROC curves were conducted to determine the cut-off, sensitivity and specificity of the risk factors which could potentially predict the onset of HUA in the 2009 cohort and 2019 cohort. The area under curves (AUC) of BMI were presented in Figure 2. As demonstrated by the ROC curves, BMI $24.48 \mathrm{~kg} / \mathrm{m}^{2}$ displayed good capacities to discriminate HUA from nonHUA in the 2009 cohort (AUC $=0.722, p<0.01,95 \% \mathrm{Cl} 0.628-0.849$ ), while BMI $23.84 \mathrm{~kg} / \mathrm{m}^{2}$ could predict the onset of HUA in the 2019 cohort (AUC= $0.679, p<0.01,95 \% \mathrm{Cl} 0.667-0.691)$.

\section{Discussion}

Our research is the first study on the changes in prevalence of HUA in Eastern China in recent decade. In this survey, the prevalence of HUA in the 2009 cohort and 2019 cohort were 13.3\% and 18.3\%, respectively. The total prevalence of HUA in the 2019 cohort was significantly higher than that in the 2009 cohort both in males and females. Liu et al. [27] have demonstrated that the prevalence of HUA in the Chinese population has increased rapidly from 2000 to 2014. In the United States, the prevalence of HUA has increased significantly from $18.2 \%$ in NHANES 1988-1994 to $21.4 \%$ in NHANES 2007-2008 [28]. It was reported that the prevalence of HUA among men in the United States (21.2\%) was very close to that of women (21.6\%) [28]. This is very different from our findings. We found that the prevalence of HUA among men in the Chinese population was significantly higher than that in women.

In addition, our data indicated the prevalence rates of HUA in both males and females in 2019 were significantly higher than those in 2009 (males: $26.4 \%$ vs. $17.3 \%$; females: $7.5 \%$ vs. $4.5 \%$ ). Previous studies have reported similar results in other regions of China [29] and 
in other countries such as Japan and Bangladesh [30,31]. There was obvious gender difference in the prevalence of HUA in the 2009 and 2019 cohort, and the prevalence of HUA in men was much higher than that in women. The reason may be related to differences in estrogen levels, eating habits and weight gain between men and women. Women, influenced by traditional Chinese culture, tend to take on more housework and may also deliberately control weight to maintain their figures. Men with extensive social networks usually accept a high-fat diets or alcohol. Although the prevalence rates of HUA in females aged 20-39 years old were significantly higher in the 2019 cohort than 2009 cohort, there was no significant difference of HUA prevalence in females aged 40-79 years old between 2009 and 2019. The prevalence of HUA in females was gradually increased with age starting from the age of 40 both in 2009 and 2019.

HUA is generally considered to be a common disease in middle-aged men, and males aged 40-49 years old was indeed the main population with HUA in 2009. However, in 2019, the prevalence of HUA in young men was higher than that of middle-aged males. Among them, the prevalence of HUA in young men aged 20-29 years old in the 2019 cohort (31.92\%) was nearly three times of that in males of the same age group in the 2009 cohort (10.04\%). Therefore, we suggest that young men are the group with the fastest increase in the prevalence of HUA in recent 10 years. In 2019, young men have replaced middle-aged men as the main population with HUA. This condition may be due to unhealthy lifestyles in young men, including high-fat diet and lack of physical exercise, which are considered to be an important determinant of elevated serum UA levels. The high prevalence of HUA not only induces a high risk of gout [32], but is also associated with increased risks of hypertension, diabetes, CVD and CKD [33-35]. The rising prevalence of HUA among young men suggests that the prevalence of gout and other related metabolic disorders is also increasing. Therefore, special attention should be paid to the prevention and treatment of HUA in young males.

In our study, we also found that BMI was significantly associated with the onset of HUA both in the 2009 cohort and 2019 cohort. Obesity has become a global problem and is recognized as a risk factor leading to multiple adverse health consequences [10]. Several studies have demonstrated the relationship between obesity and HUA [31, 36]. A positive correlation between serum UA level and obesity was found in Bangladeshi adults [31]. A Japanese study reported that HUA was significantly associated with central obesity in obese populations rather than in normal-weight men and women [30]. In the Chinese population, obesity was found to be a crucial risk factor for HUA in females [36]. Therefore, our results were consistent with the previous findings. In addition, we found that BMI $124.48 \mathrm{~kg} / \mathrm{m}^{2}$ and BMI $23.84 \mathrm{~kg} / \mathrm{m}^{2}$ could display good capacities to discriminate patients with HUA from non-HUA in the 2009 and 2019 cohort, respectively. This result further confirmed the association between HUA and obesity. Nevertheless, we should notice that the cut-off of BMI used to predict the onset of HUA has begun to decline in the past decade. This may imply that the people with a lower BMI than before are also likely to suffer from HUA, but the reason for this change is still unclear. It may be related to changes in diet and lifestyles to a certain extent. For example, a recent study showed that the Chinese diet was shifting to high-fat and high-energy-dense foods [37]. The consumption of vegetable oils, animal-derived foods and processed foods that are rich in refined starch, sugar, salt and unhealthy fats continued to increase. The consumption of staple foods has gradually changed from the traditional staple foods of coarse grains to refined cereals [38]. Moreover, according to the Global Burden of Diseases database, the burden of disease in urban areas of China, which are associated with individual behaviors and habits such as unhealthy diet, drinking, smoking and lack of physical exercise, is steadily increasing [39].

Our research also has several limitations. Firstly, all subjects were from a single center, which may limit the extrapolation of our conclusions. Secondly, most of the elderly who participated in this study were in good health, and patients with serious illnesses were unable to participate in our study, which may lead to an underestimation of the prevalence of HUA among the elderly. Thirdly, the drinking history, smoking history and previous medical history of these subjects were not obtained, which may also affect the results of our study.

\section{Conclusions}

1) The prevalence rate of HUA in Eastern China has been increasing rapidly in the past 10 years. 2) The population with the highest HUA prevalence rate in 2019 was young men aged 20-29 years, while the population with the peak prevalence rate in 2009 was middle-aged men (40-49 years old). 3) BMI was positively correlated with the occurrence of HUA, and BMI displayed a good capacity to discriminate the patients with HUA from non-HUA both in the 2009 cohort and 2019 cohort.

\section{Abbreviations}

HUA

Hyperuricemia

UA 
Uric acid

BMI

Body mass index

$\mathrm{BH}$

Body height

BW

Body weight

SBP

Systolic blood pressure

DBP

Diastolic blood pressure

FBG

Fasting blood-glucose

TG

Triglyceride

$\mathrm{CHOL}$

Cholesterol

HDL

High-density lipoprotein

LDL

Low-density lipoprotein

$\mathrm{Cr}$

Creatinine

BUN

Blood urea nitrogen

Cl

Confidence interval

CVD

Cardiovascular disease

CKD

Chronic kidney disease

SPSS

Statistical package for social sciences

ROC

Receiver-operating characteristic

AUC

Area under curves

\section{Declarations}

\section{Acknowledgements}

Not applicable.

\section{Authors' contributions}

Conceived and designed the research, wrote and revised the manuscript: DMS, YZ, YX and ZWZ. Collected the data: DMS, SYF, YLW, JL, YL, JX and SCX. Analyzed the data: DMS, NL and YX. All authors approved the final manuscript as submitted and agreed to be accountable for all aspects of the work.

\section{Funding}

This study was sponsored by Psychosomatic Medicine Project of Key Developing Disciplines of Shanghai Municipal Health Commission (2019ZB0202), the National Natural Science Foundation of China (Grant No. 81974105), Novo Nordisk China Diabetes 
Young Scientific Talent Research Funding, Shanghai Pujiang Program (Grant No. 2019PJD050), Cultivation project for National Natural Science Foundation of Shanghai Tongji Hospital, Jiangsu TCM Science and Technology Development Plan (Grant No. YB2020087), and Doctoral Fund of Northern Jiangsu Hospital (Grant No. BSQDJ0142).

\section{Availability of data and materials}

The datasets used and analysed during the current study are available from the corresponding author on reasonable request.

\section{Ethics approval and consent to participate}

All procedures performed in studies involving human participants were in accordance with the 1964 Helsinki declaration and its later amendments or comparable ethical standards. Ethical approval for the study was obtained from the Ethical Committee of Northern Jiangsu People's Hospital. Informed consent was obtained from each individual participant included in the study.

\section{Consent for publication}

Not applicable.

\section{Competing interests}

The authors declare that they have no conflict of interest.

\section{Acknowledgements}

All of the authors declare that they have no competing interests.

\section{References}

1. Kimiyoshi Ichida, Hirotaka Matsuo, Tappei Takada, Akiyoshi Nakayama, Keizo Murakami, Toru Shimizu, et al. Decreased extra-renal urate excretion is a common cause of hyperuricemia. Nature communications. 2012; 3: 764. https://doi.org/10.1038/ncomms1756.

2. Junxia Su, Yuhui Wei, Minglong Liu, Tianxi Liu, Jianhua Li, Yuanchun Ji, et al. Anti-hyperuricemic and nephroprotective effects of Rhizoma Dioscoreae septemlobae extracts and its main component dioscin via regulation of mOAT1, mURAT1 and mOCT2 in hypertensive mice. Arch Pharm Res. 2014; 37 (10): 1336-1344. https://doi.org/10.1007/s12272-014-0413-6.

3. Xiaokang Dong, Honglei Zhang, Fang Wang, Xiaotian Liu, Kaili Yang, Runqi Tu, et al. Epidemiology and prevalence of hyperuricemia among men and women in Chinese rural population: The Henan Rural Cohort Study. Mod Rheumatol. 2019; 30(5): 910-920. https://doi.org/10.1080/14397595.2019.1660048.

4. Georgiana Cabău, Tania O Crișan, Viola Klück, Radu A Popp, Leo A B Joosten. Urate-induced immune programming: Consequences for gouty arthritis and hyperuricemia. Immunol Rev. 2020; 294(1):92-105. https://doi.org/10.1111/imr.12833.

5. Ravi K Narang, Greg G Gamble, Ruth Topless, Murray Cadzow, Lisa K Stamp, Tony R Merriman, et al. Assessing the Relationship Between Serum Urate and Urolithiasis Using Mendelian Randomization: An Analysis of the UK Biobank. Am J Kidney Dis. 2021; 78 (20): 210-218. https://doi.org/10.1053/j.ajkd.2020.11.018.

6. Richard J Johnson, Takahiko Nakagawa, Diana Jalal, Laura Gabriela Sánchez-Lozada, Duk-Hee Kang, Eberhard Ritz. Uric acid and chronic kidney disease: which is chasing which? Nephrol Dial Transplant. 2013; 28 (9): 2221-2228. https://doi.org/10.1093/ndt/gft029.

7. Abbas Dehghan, Mandy van Hoek, Eric J G Sijbrands, Albert Hofman, Jacqueline C M Witteman. High serum uric acid as a novel risk factor for type 2 diabetes. Diabetes care. 2008; 31(2):361-362. https://doi.org/10.2337/dc07-1276.

8. Peter C Grayson, Seo Young Kim, Michael LaValley, Hyon K Choi. Hyperuricemia and incident hypertension: a systematic review and meta-analysis. Arthritis Care Res (Hoboken). 2011; 63(1):102-110. https://doi.org/ 10.1002/acr.20344.

9. Seo Young Kim, James P Guevara, Kyoung Mi Kim, Hyon K Choi, Daniel F Heitjan, Daniel A Albert. Hyperuricemia and coronary heart disease: a systematic review and meta-analysis. Arthritis Care Res (Hoboken). 2010; 62(2):170-180.

https://doi.org/10.1002/acr.20065.

10. Luxia Zhang, Fang Wang, Li Wang, Wenke Wang, Bicheng Liu, Jian Liu, et al. Prevalence of chronic kidney disease in China: a crosssectional survey. Lancet, 2012; 379 (9818): 815-822. https://doi.org/10.1016/S0140-6736(12)60033-6. 
11. Dongwang Qi, Jie Liu, Conglin Wang, Lixia Wang, Xinxin Zhang, Qiuxing Lin, et al. Sex-specific differences in the prevalence of and risk factors for hyperuricemia among a low-income population in China: a cross-sectional study. Postgrad Med. 2020; 132 (6): $559-$ 567. https://doi.org/10.1080/00325481.2020.1761133.

12. Zifeng Liu, Xiaoting Su, Mianli Xiao, Peien Zhou, Jianwei Guo, Yixiang Huang, et al. Association between Eating Away from Home and Hyperuricemia: A Population-Based Nationwide Cross-Sectional Study in China. Biomed Res Int. 2019; 2019: 2792681. eCollection. https://doi.org/10.1155/2019/2792681.

13. Hong Liu, Xiao-Min Zhang, Yan-Li Wang, Bi-Cheng Liu. Prevalence of hyperuricemia among Chinese adults: a national crosssectional survey using multistage, stratified sampling. J Nephrol. 2014; 27 (6): 653-658. https://doi.org/10.1007/s40620-014-0082z.

14. R Wang, Z Tang, F Sun, LJ Diao. Prevalence of hyperuricemia in the elderly in 7 areas of China. Zhonghua Liu Xing Bing Xue Za Zhi. 2018; 39 (3): 286-288. https://doi.org/10.3760/cma.j.issn.0254-6450.2018.03.007.

15. ML Zheng, YH Lai, He XN, XW Tan. Correlation of detection of hyperuricemia with hypertension in healthy population in Guangzhou City. China Trop Med. 2008; 8: 512-514.

16. XH Han, XY Yao, XS Fang. The prevalence of hyperuricemia and abnormal of lipid and glucose in medical examination population. Shanxi Med J. 2008; 37: 438-439.

17. YJ Cao, Y Liu, T Li, BS Yan, ZZ Wang, XL Yang. Investigation of the increasing of policemen in blood uric acid and triglyceride in Haikou. Hainan Med J. 2010; 21: 120-121.

18. CJ Gao, YG Jiang, ZL Tang. Epidemiological analysis of hyperuricemia in 39824 healthy subjects in Anhui province. J Pract Med. 2008; 24; 20: 3589-3590.

19. JM Zhang, LN Yao, H Li. Study on hyperuricemia in the physical examination population in Zhengzhou area. J Zhengzhou University. 2009; 44; 1 : 174-175.

20. Qingxi Zhang, Hongqiang Gong, Chutong Lin, Qiang Liu, Yangjin Baima, Yunting Wang, et al. The prevalence of gout and hyperuricemia in middle-aged and elderly people in Tibet Autonomous Region, China: A preliminary study. Medicine (Baltimore). 2020; 99 (2): e18542. https://doi.org/10.1097/MD.0000000000018542.

21. Ming-Yun Chen, Cui-Chun Zhao, Ting-Ting Li, Yue Zhu, Tian-Pei Yu, Yu-Qian Bao, et al. Serum uric acid levels are associated with obesity but not cardio-cerebrovascular events in Chinese inpatients with type 2 diabetes. Sci Rep. 2017; 7(1): 40009. https://doi.org/10.1038/srep40009.

22. Cuiling Zhu, Ran Cui, Mingming Gao, Sharvan Rampersad, Hui You, Chunjun Sheng, et al. The Associations of Serum Uric Acid with Obesity-Related Acanthosis nigricans and Related Metabolic Indices. Int J Endocrinol. 2017; 2017: 5438157. https://doi.org/10.1155/2017/5438157.

23. Ying Duan, Wei Liang, Lijun Zhu, Ting Zhang, Linghong Wang, Zhognhua Nie, et al. Association between serum uric acid levels and obesity among university students (China). Nutr Hosp. 2015; 31(6): 2407-2411. https://doi.org/10.3305/nh.2015.31.6.8734.

24. Chao Yang, Shujuan Yang, Chunhong Feng, Chuan Zhang, Weiwei Xu, Liyun Zhang, et al. Associations of hyperuricemia and obesity with remission of nonalcoholic fatty liver disease among Chinese men: A retrospective cohort study. PloS one. 2018; 13(2): e0192396. https://doi.org/10.1371/journal.pone.0192396.

25. G Neil Thomas, Sai-Yin Ho, Edward D Janus, Karen S L Lam, Anthony J Hedley, Tai Hing Lam. Hong Kong Cardiovascular Risk Factor Prevalence Study Steering Committee, The US National Cholesterol Education Programme Adult Treatment Panel III (NCEP ATP III) prevalence of the metabolic syndrome in a Chinese population. Diabetes Res Clin Pract. 2005; 67(3): 251-257. https://doi.org/10.1016/j.diabres.2004.07.022.

26. Bei-Fan Zhou. Predictive values of body mass index and waist circumference for risk factors of certain related diseases in Chinese adults: study on optimal cut-off points of body mass index and waist circumference in Chinese adults. Biomed Environ Sci. 2005; 15 (1):83-96. PMID: 12046553.

27. Rui Liu, Cheng Han, Di Wu, Xinghai Xia, Jianqiu Gu, Haixia Guan, et al. Prevalence of Hyperuricemia and Gout in Mainland China from 2000 to 2014: A Systematic Review and Meta-Analysis. BioMed Res Int. 2015; 2015: 762820. https://doi.org/10.1155/2015/762820.

28. Katrine L Wallace, Aylin A Riedel, Nancy Joseph-Ridge, Robert Wortmann. Increasing prevalence of gout and hyperuricemia over 10 years among older adults in a managed care population. J Rheumatol.2004; 31(8): 1582-1527. PMID: 15290739.

29. Q Wang, C Wang, J Xue, M M Chen, H W Sun, M Jiang. Characteristics of serum uric acid distribution in occupation, age, gender groups and its influencing factors in physical examination subjects in Nanjing from 2012 to 2016. Zhonghua Nei Ke Za Zhi. 2021; 
60(1): 29-34. https://doi.org/10.3760/cma.j.cn112138-20200306-00198.

30. Takako Shirasawa, Hirotaka Ochiai, Takahiko Yoshimoto, Satsue Nagahama, Akihiro Watanabe, Reika Yoshida, et al. Crosssectional study of associations between normal body weight with central obesity and hyperuricemia in Japan. BMC Endocr Disord. 2020; 20(1): 2. https://doi.org/10.1186/s12902-019-0481-1.

31. Nurshad Ali, Rasheda Perveen, Shahnaz Rahman, Shakil Mahmood, Sadaqur Rahman, Shiful Islam, et al. Prevalence of hyperuricemia and the relationship between serum uric acid and obesity: A study on Bangladeshi adults. PLoS One. 2018; 13(11): e0206850. https://doi.org/10.1371/journal.pone.0206850.

32. Somchai Uaratanawong, S Suraamornkul, S Angkeaw, R Uaratanawong. Prevalence of hyperuricemia in Bangkok population. Clin Rheumatol. 2011; 30 (7): 887-893. https://doi.org/10.1007/s10067-011-1699-0.

33. Vitool Lohsoonthorn, Bodi Dhanamun, Michelle A Williams. Prevalence of hyperuricemia and its relationship with metabolic syndrome in Thai adults receiving annual health exams. Arch Med Res. 2016; 37(7):883-889. https://doi.org/10.1016/j.arcmed.2006.03.008.

34. Ismail Sari, Servet Akar, Betul Pakoz, Ali Riza Sisman, Oguz Gurler, Merih Birlik, et al. Hyperuricemia and its related factors in an urban population, Izmir, Turkey. Rheumatol Int. 2009; 29(8):869-874. https://doi.org/10.1007/s00296-008-0806-2.

35. Yanyan Zhu, Bhavik J Pandya, Hyon K Choi. Prevalence of gout and hyperuricemia in the US general population: the National Health and Nutrition Examination Survey 2007-2008. Arthritis Rheum. 2011; 63(10):3136-3141. https://doi.org/10.1002/art.30520.

36. Peige Song, He Wang, Wei Xia, Xinlei Chang, Manli Wang, Lin An. Prevalence and correlates of hyperuricemia in the middle-aged and older adults in China. Sci Rep. 2018; 8(1): 4314. https://doi.org/10.1038/s41598-018-22570-9.

37. Xiao Chang, Ruth S DeFries, Liming Liu, Kyle Davis. Understanding dietary and staple food transitions in China from multiple scales. Plos One. 2018; 13(4): e0195775. https://doi.org/10.1371/journal.pone.0195775.

38. Aixi Han, Tianhao Sun, Jing Ming, Li Chai, Xiawei Liao. Are the Chinese Moving toward a Healthy Diet? Evidence from Macro Data from 1961 to 2017. Int J Environ Res Public Health. 2020; 17(15):5294. https://doi.org/10.3390/ijerph17155294.

39. Xinhu Li, Jinchao Song, Tao Lin, Jane Dixon, Guoqin Zhang, Hong Ye. Urbanization and health in China, thinking at the national, local and individual levels. Environ Health. 2016; 15(S1): 32. https://doi.org/10.1186/s12940-016-0104-5.

\section{Tables}

Due to technical limitations, table 2 is only available as a download in the Supplemental Files section.

\section{Figures}


A

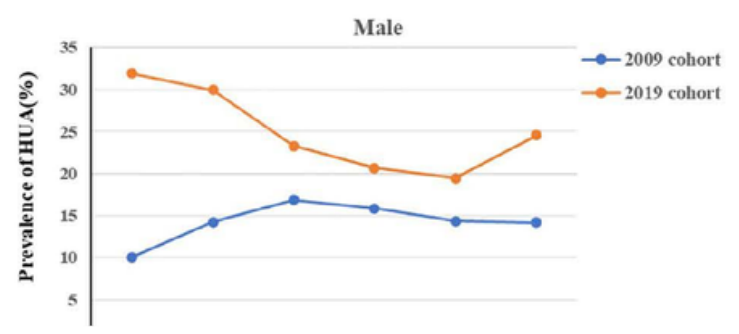

B

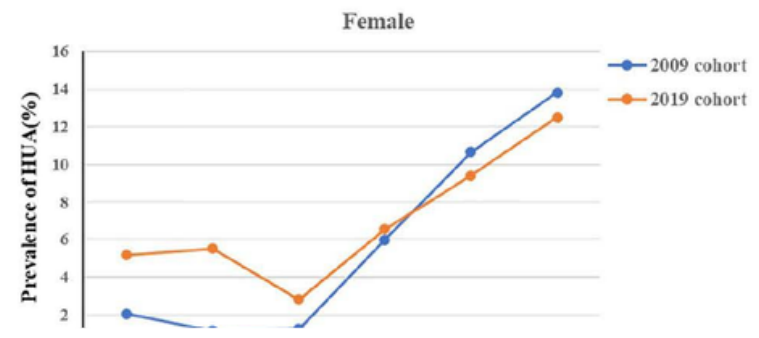

\section{Figure 1}

The prevalence of HUA of males and females in different age groups and BMI groups in 2009 and 2019 cohorts. 


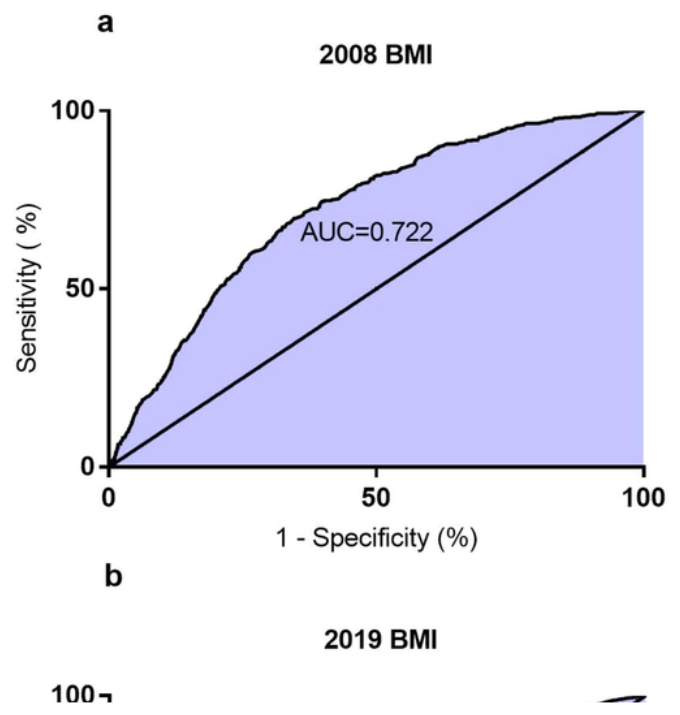

\section{Figure 2}

Receiver-operating characteristic (ROC) curves for the ability of BMI to discriminate the HUA patients from the non-HUA participants in 2009 cohort and 2019 cohort.

The area under the ROC curve (AUC) $=0.722$ for BMI in 2009 cohort, $P<0.001,95 \% \mathrm{Cl} 0.701-0.743$; AUC= 0.679 for BMI in 2019 cohort, $P$ $<0.001,95 \% \mathrm{Cl} 0.667-0.691$.

\section{Supplementary Files}

This is a list of supplementary files associated with this preprint. Click to download.

- Table2.docx 